\title{
ANALYSIS AND SLIDING CONTROLler DESIGN FOR HYBRID SYNCHRONIZATION OF HYPERCHAOTIC YUJUN SYSTEMS
}

\author{
Sundarapandian Vaidyanathan \\ Research and Development Centre, Vel Tech University \\ Avadi, Chennai-600 062, Tamil Nadu, INDIA \\ sundarvtuegmail.com
}

\begin{abstract}
Hybrid synchronization of chaotic systems is a research problem with a goal to synchronize the states of master and slave chaotic systems in a hybrid manner, namely, their even states are completely synchronized (CS) and odd states are anti-synchronized. This paper deals with the research problem of hybrid synchronization of chaotic systems. First, a detailed analysis is made on the qualitative properties of hyperchaotic Yujun system (2010). Then sliding controller has been derived for the hybrid synchronization of identical hyperchaotic Yujun systems, which is based on a general hybrid result derived in this paper. $M A T L A B$ simulations have been shown in detail to illustrate the new results derived for the hybrid synchronization of hyperchaotic Yujun systems. The results are proved using Lyapunov stability theory.
\end{abstract}

\section{KEYWORDS}

Hyperchaotic Yujun System, Hyperchaos, Sliding Control, Synchronization, Lyapunov Stability Theory.

\section{INTRODUCTION}

By definition, a hyperchaotic system is a chaotic system with more than one positive Lyapunov exponent which implies that the dynamics can be expanded in several directions.

In 1979, Rössler [1] discovered the hyperchaotic system. Hyperchaotic systems have found many research applications in neural networks [2], secure communications [3], etc.

In the chaos literature, there has been active interest in developing new methodology for synchronization of chaotic and hyperchaotic systems.

Some important methods developed for chaos synchronization are OGY method [4], PC method [5], sampled-data feedback method [6], time-delay feedback method [7], active control method [8-10], adaptive control method [11-13], backstepping design method [14-15] and sliding mode control method [16-18].

In this paper, a qualitative analysis has been presented for the 4-D hyperchaotic Yujun system ([19], 2010). Next, a sliding mode controller has been derived for the hybrid synchronization of identical hyperchaotic Yujun systems. 
The organization of this paper can be detailed as follows. Section 2 contains general sliding control results for the hybrid synchronization of master and slave systems. Section 3 describes the hyperchaotic Yujun system. Section 4 describes the sliding controller design for the hybrid synchronization of hyperchaotic Yujun systems and MATLAB simulations.

As the drive system, we take the chaotic system

$$
\dot{x}=A x+f(x)
$$

where $x \in R^{n}$ is the system state, $A$ is an $n \times n$ constant matrix and $f: R^{n} \rightarrow R^{n}$ is the nonlinear part.

As the response system, we take the identical controlled chaotic system

$$
\dot{y}=A y+f(y)+u
$$

where $y \in R^{n}$ is the system state and $u \in R^{m}$ is the sliding controller to be constructed.

A formal definition of the hybrid synchronization error between the drive system (1) and the response system (2) can be provided as

$$
e_{i}=\left\{\begin{array}{cc}
y_{i}-x_{i}, & \text { when } i \text { is an odd integer } \\
y_{i}+x_{i}, & \text { when } i \text { is an even integer }
\end{array}\right.
$$

Differentiating (3) and simplifying, we obtain the error dynamics as

$$
\dot{e}=A e+\eta(x, y)+u,
$$

The design goal is to determine $u(t)$ so as to drive the error to zero, i.e.

$$
\lim _{t \rightarrow \infty}\|e(t)\|=0 \quad \text { for each } e(0) \in R^{n} .
$$

As methodology, we use sliding mode control (SMC) to solve the above design problem.

First, we define the controller $u$ as

$$
u=-\eta(x, y)+B v
$$

where $B$ is chosen carefully such that $(A, B)$ is completely controllable.

If we substitute (5) into (4), then the error dynamics takes the simple form

$$
\dot{e}=A e+B v
$$

Eq. (7) represents a single-input linear time-invariant control system.

In SMC control design, we take the sliding variable as

$$
s(e)=C e=c_{1} e_{1}+c_{2} e_{2}+\cdots+c_{n} e_{n}
$$


where $C=\left[\begin{array}{llll}c_{1} & c_{2} & \cdots & c_{n}\end{array}\right]$ is a constant vector to be determined.

In SMC design, the error system (7) is confined to the sliding manifold defined by

$$
S=\left\{x \in R^{n} \mid s(e)=0\right\}
$$

The sliding control theory demands that the manifold $S$ be invariant under the flow of (7).

Thus, the error system (7) must satisfy the following two conditions, viz.

$$
s(e)=0
$$

and

$$
\dot{s}(e)=0
$$

By making use of Eqns. (7) and (8), Eq. (10) can be simplified in an equivalent form as

$$
\dot{s}(e)=C[A e+B v]=0
$$

Next, we find the solution $v$ of the equation (11). Thus, we get the equivalent control law

$$
v_{\mathrm{eq}}(t)=-(C B)^{-1} C A e(t)
$$

In Eq. (12), $C$ is selected such that $C B$ is a non-zero scalar.

Plugging in the equivalent control law (12) into (7) yields the simplified dynamics

$$
\dot{e}=\left[I-B(C B)^{-1} C\right] A e
$$

We choose $C$ so that the system matrix $\left[I-B(C B)^{-1} C\right] A$ is stable.

It is noted that the resulting system (13) is GAS (globally asymptotically stable).

In our sliding control methodology, to obtain the SMC control for (7), we make use of the constant plus proportional rate law. This is given by the equation

$$
\dot{s}=-q \operatorname{sgn}(s)-k s
$$

where the gains $q>0, k>0$ are constants.

We shall find the gains so that the following hold:

(1) The sliding condition is satisfied.

(2) The sliding motion will occur.

By the use of equations (11) and (14), we get the sliding control $v(t)$ as 
International Journal of Computer Science, Engineering and Applications (IJCSEA) Vol.3, No.4, August 2013

$$
v(t)=-(C B)^{-1}[C(k I+A) e+q \operatorname{sgn}(s)]
$$

Theorem 1. The drive system (1) and the response system (2), which are identical chaotic systems, are globally and asymptotically hybrid synchronized for all initial conditions $x(0), y(0) \in R^{n}$ by the sliding control law

$$
u(t)=-\eta(x, y)+B v(t)
$$

where $v(t)$ is given by $(15)$ and $B$ is chosen such that $(A, B)$ is controllable.

Proof. We prove this theorem by applying Lyapunov stability theory [20].

First, we make a substitution of (16) and (15) into the error system (4).

After this substitution, a simple calculation shows that the error dynamics reduces to

$$
\dot{e}=A e-B(C B)^{-1}[C(k I+A) e+q \operatorname{sgn}(s)]
$$

As we prove using Lyapunov stability theory, we have to construct a Lyapunov function for the error dynamics (17). We consider the following function as a candidate, viz.

$$
V(e)=\frac{1}{2} s^{2}(e)
$$

We note that $V$ is a positive definite function on $R^{n}$.

Next, we find the time derivative of $V$ along the trajectories of (16) or the equivalent dynamics (14). Thus, we obtain

$$
\dot{V}(e)=s(e) \dot{s}(e)=-k s^{2}-q \operatorname{sgn}(s) s
$$

We note that $\dot{V}$ is a negative definite function on $R^{n}$.

Hence, the proof is complete by Lyapunov stability theory [20].

\section{ANALYSIS OF HyPERCHAOTIC YuJUn SYSTEM}

The hyperchaotic Yujun system ([19], 2010) is a four-dimensional system given by

$$
\begin{aligned}
& \dot{x}_{1}=a\left(x_{2}-x_{1}\right)+x_{2} x_{3} \\
& \dot{x}_{2}=c x_{1}-x_{2}+x_{4}-x_{1} x_{3} \\
& \dot{x}_{3}=-b x_{3}+x_{1} x_{2} \\
& \dot{x}_{4}=r x_{4}-x_{1} x_{3}
\end{aligned}
$$

where $a, b, c, r$ are positive parameters and $x_{1}, x_{2}, x_{3}, x_{4}$ are the states of the system.

The system (20) is hyperchaotic when the parameter values are chosen as 
International Journal of Computer Science, Engineering and Applications (IJCSEA) Vol.3, No.4, August 2013

$$
a=35, b=8 / 3, c=55 \text { and } r=1.3
$$

When the parameters are given by (21), the system Lyapunov exponents are obtained as

$$
L_{1}=1.4164, \quad L_{2}=0.5318, \quad L_{3}=0, \quad L_{4}=-39.1015 \text {. }
$$

Since there are two positive Lyapunov exponents, the above calculation confirms that the system (20) is hyperchaotic when the parameter values are given by (21).

The projections of the hyperchaotic Yujun attractor are shown in Figure 1.
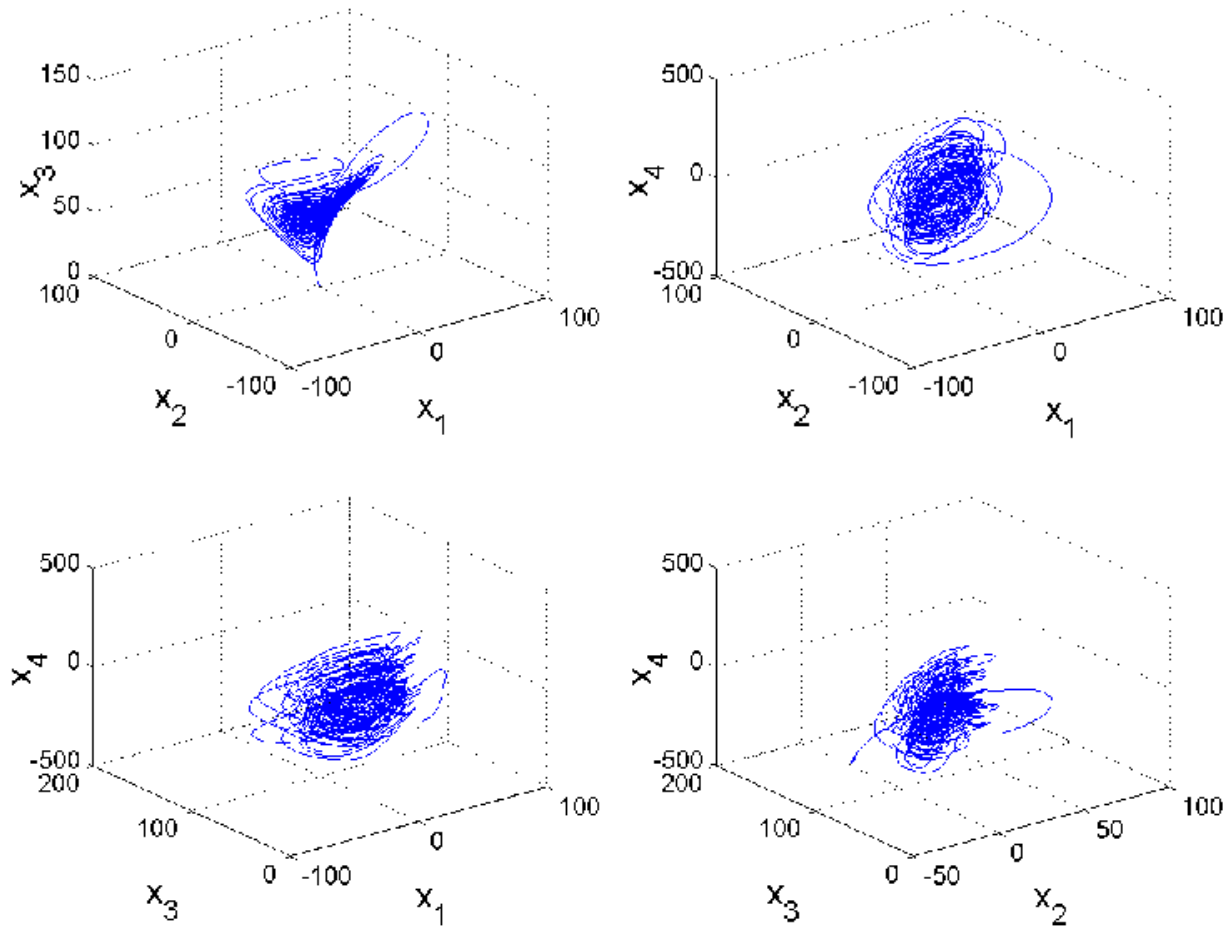

Figure 1. The Projections of the Hyperchaotic Yujun Attractor

\section{SMC DESIGn For HybRID SynChronization OF HyPERCHAOTIC YUJUN SYSTEMS}

In this section, we take the drive system as the hyperchaotic Yujun system given by

$$
\begin{aligned}
& \dot{x}_{1}=a\left(x_{2}-x_{1}\right)+x_{2} x_{3} \\
& \dot{x}_{2}=c x_{1}-x_{2}+x_{4}-x_{1} x_{3} \\
& \dot{x}_{3}=-b x_{3}+x_{1} x_{2} \\
& \dot{x}_{4}=r x_{4}-x_{1} x_{3}
\end{aligned}
$$


where $a, b, c, r$ are positive, constant parameters of the system.

The response system is described by the controlled hyperchaotic Yujun system as

$$
\begin{aligned}
& \dot{y}_{1}=a\left(y_{2}-y_{1}\right)+y_{2} y_{3}+u_{1} \\
& \dot{y}_{2}=c y_{1}-y_{2}+y_{4}-y_{1} y_{3}+u_{2} \\
& \dot{y}_{3}=-b y_{3}+y_{1} y_{2}+u_{3} \\
& \dot{y}_{4}=r y_{4}-y_{1} y_{3}+u_{4}
\end{aligned}
$$

where $u_{1}, u_{2}, u_{3}, u_{4}$ are the sliding mode controllers to be designed using the systematic procedure outlined in Section 2.

The hybrid synchronization error is given by the definition

$$
\begin{aligned}
& e_{1}=y_{1}-x_{1} \\
& e_{2}=y_{2}+x_{2} \\
& e_{3}=y_{3}-x_{3} \\
& e_{4}=y_{4}+x_{4}
\end{aligned}
$$

A simple workout yields the dynamics of (25) as

$$
\begin{aligned}
& \dot{e}_{1}=a\left(e_{2}-e_{1}\right)-2 a e_{2}+u_{1} \\
& \dot{e}_{2}=4 e_{1}+c e_{2}+4 e_{4}+8 x_{1}-10\left(y_{1} y_{3}+x_{1} x_{3}\right)+u_{2} \\
& \dot{e}_{3}=-b e_{3}+y_{2}^{2}-x_{2}^{2}+u_{3} \\
& \dot{e}_{4}=-d e_{1}-2 d x_{1}+u_{4}
\end{aligned}
$$

We write the error dynamics (26) in a matrix form.

Thus, we get the system

$$
\dot{e}=A e+\eta(x, y)+u
$$

In Eq. (27), the associated matrix and vectors are

$$
A=\left[\begin{array}{cccc}
-a & a & 0 & 0 \\
4 & c & 0 & 4 \\
0 & 0 & -b & 0 \\
-d & 0 & 0 & 0
\end{array}\right], \quad \eta(x, y)=\left[\begin{array}{c}
-2 a x_{2} \\
8 x_{1}-10\left(y_{1} y_{3}+x_{1} x_{3}\right) \\
y_{2}^{2}-x_{2}^{2} \\
-2 d x_{1}
\end{array}\right] \text { and } u=\left[\begin{array}{l}
u_{1} \\
u_{2} \\
u_{3} \\
u_{4}
\end{array}\right]
$$

Next, we carry out the sliding controller design for the hybrid synchronization of the hyperchaotic systems considered in this paper.

For this paper, we first define $u$ as

$$
u=-\eta(x, y)+B v
$$


In (29), the matrix $B$ is chosen such that $(A, B)$ is controllable.

A simple selection for $B$ is

$$
B=\left[\begin{array}{l}
1 \\
1 \\
1 \\
1
\end{array}\right]
$$

We take the parameter values of the hyperchaotic Yujun system as

$$
a=35, b=3, c=21 \text { and } d=2
$$

We also take the sliding mode variable as

$$
s=C e=\left[\begin{array}{llll}
-1 & -2 & 0 & 1
\end{array}\right] e=-e_{1}-2 e_{2}+e_{4}
$$

The choice of $C$ renders the system globally asymptotically stable in the sliding mode.

We take the sliding mode gains $k$ and $q$ as

$$
k=6 \text { and } q=0.2
$$

By making use of Eq. (15), we derive the sliding control $v(t)$ as

$$
v(t)=9.5 e_{1}-44.5 e_{2}-e_{4}+0.1 \operatorname{sgn}(s)
$$

Finally, for the hybrid synchronization of hyperchaotic Yujun systems, we get the sliding control law given by the equation

$$
u=-\eta(x, y)+B v
$$

Theorem 2. The identical hyperchaotic Yujun systems (23) and (24) are asymptotically hybrid synchronized for all initial conditions by implementing the sliding mode controller udefined by (34).

\subsection{Numerical Results}

The initial values of the master system (23) are taken as

$$
x_{1}(0)=20, x_{2}(0)=-6, x_{3}(0)=15, x_{4}(0)=26
$$

and the initial values of the slave system (24) are taken as

$$
y_{1}(0)=-4, y_{2}(0)=26, y_{3}(0)=-11, y_{4}(0)=28
$$

Figure 2 depicts the hybrid synchronization of the identical hyperchaotic Yujun systems. 
International Journal of Computer Science, Engineering and Applications (IJCSEA) Vol.3, No.4, August 2013

Figure 3 depicts the time history of the synchronization error states $e_{1}(t), e_{2}(t), e_{3}(t), e_{4}(t)$.
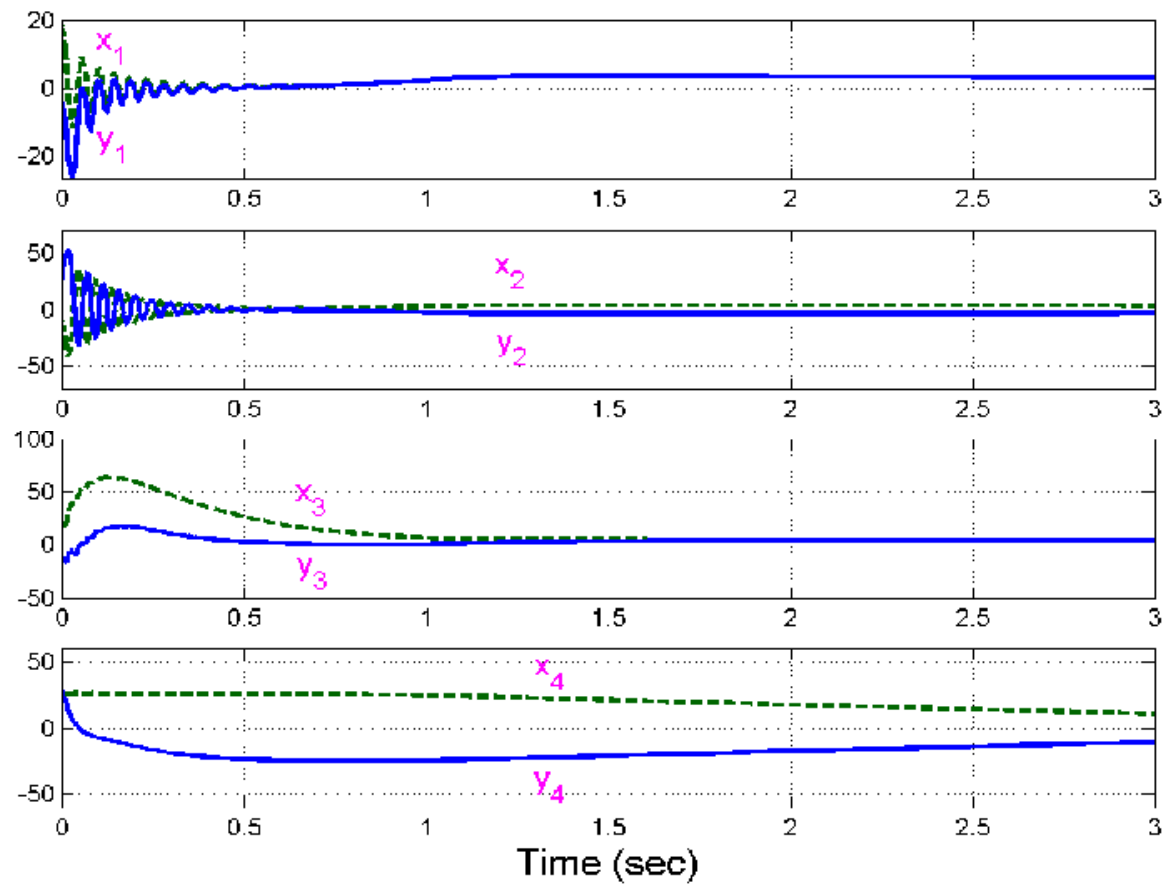

Figure 2. Hybrid Synchronization of Identical Hyperchaotic Yujun Systems

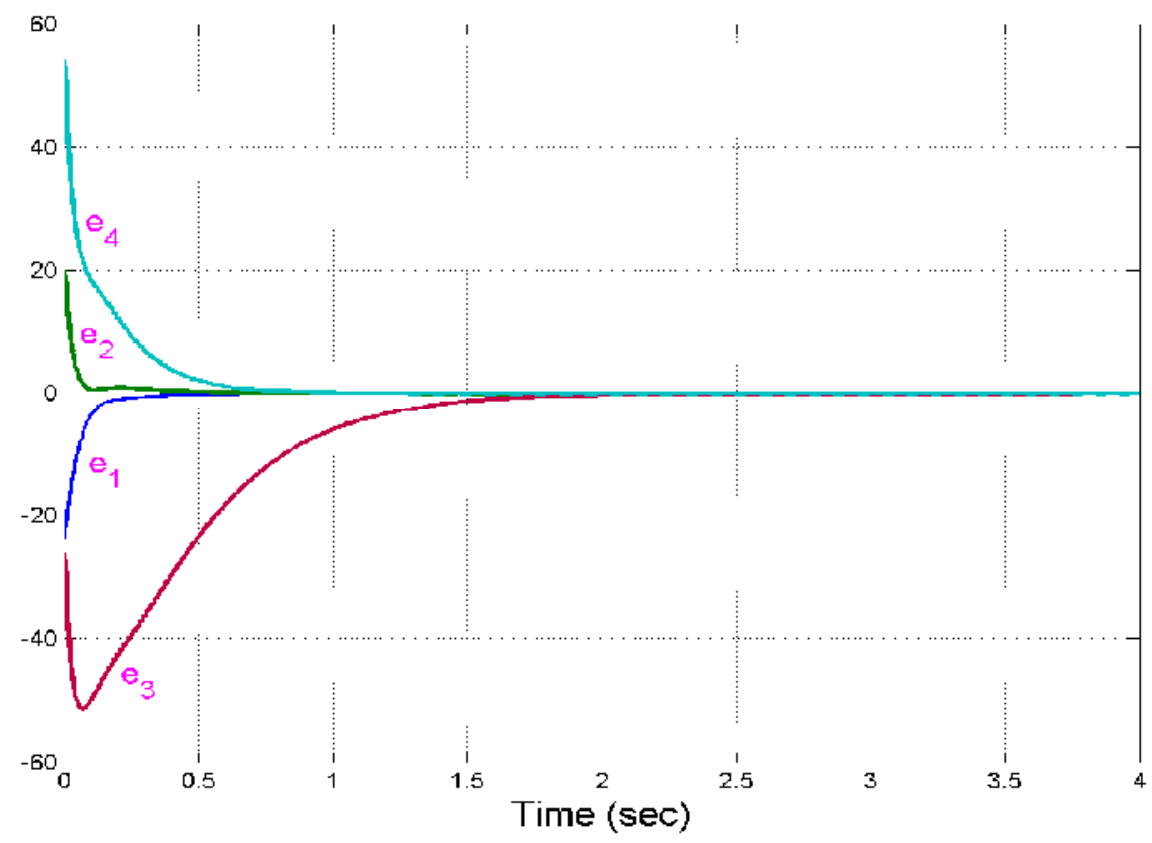

Figure 3. Time History of Error States $e_{1}(t), e_{2}(t), e_{3}(t), e_{4}(t)$ 


\section{Conclusions}

This paper investigated the hybrid synchronization problem for the hyperchaotic systems using sliding mode control (SMC) and derived some general results. Lyapunov stability theory was the methodology used for proving these results. Using this general result for hybrid synchronization, we derived a new sliding controller for achieving hybrid synchronization of the identical hyperchaotic Yujun systems (2010). MATLAB simulation results were provided to illustrate the results derived in this paper.

\section{REFERENCES}

[1] Rössler, O.E. (1979) “An equation for hyperchaos,” Phy. Lett. A, Vol. 71, pp 151-157.

[2] Huang, X., Zhao, Z., Wang, Z. \& Li, Y. (2012) "Chaos and hyperchaos in fractional-order cellular neural networks," Neurocomputing, Vol. 94, pp 13-21.

[3] Li, C., Liao, X. \& Wong, K. (2005) "Lag synchronization of hyperchaos with applications to secure communications," Chaos, Solitons \& Fractals, Vol. 23, No. 1, pp 183-193.

[4] Ott, E., Grebogi, C. \& Yorke, J.A. (1990) "Controlling chaos”, Phys. Review Lett., Vol. 64, pp 11961199.

[5] Pecora, L.M. \& Carroll, T.L. (1990) "Synchronization in chaotic systems", Phys. Review Lett., Vol. 64, pp 821-824.

[6] Wang, Y.W. \& Guan, Z.H. (2006) "Using sampled-data feedback control and linear feedback synchronization in a new hyperchaotic system," Chaos, Solitons \& Fractals, Vol. 27, pp 97-101.

[7] Kim, C.M., Kye, W.H., Rim, S. \& Lee, S.Y. (2004) "Communication key using delay times in timedelayed chaos synchronization,” Phy. Lett. A, Vol. 333, pp 235-240.

[8] Lei, Y., Xu, W. \& Zheng, H. (2005) "Synchronization of two chaotic nonlinear gyros using active control," Phy. Lett. A, Vol. 343, pp 153-158.

[9] Chen, H.H., Sheu, G.J., Lin, Y.L. \& Chen, C.S. (2009) "Chaos synchronization between two different chaotic systems via nonlinear feedback control,” Nonlinear Analysis, Vol. 70, pp 4393-4401.

[10] Sundarapandian, V. \& Karthikeyan, R. (2011) "Active controller design for global chaos antisynchronization of Li and Tigan chaotic systems," International Journal of Information Technology and Computer Science, Vol. 3, No. 4, pp 255-268.

[11] Chen, S.H. \& Lü, J. (2002) "Synchronization of an uncertain unified system via adaptive control," Chaos, Solitons \& Fractals, Vol. 14, pp 643-647.

[12] Hsu, C.F. (2011) “Adaptive fuzzy wavelet neural controller design for chaos synchronization,” Expert Systems with Applications, Vol. 38, No. 8, pp 10475-10483.

[13] Sundarapandian, V. (2011) "Adaptive synchronization of hyperchaotic Lorenz and hyperchaotic Lü systems,” International J. Instrumentation and Control Systems, Vol. 1, No. 1, pp 1-18.

[14] Wu, X. \& Lü, J. (2003) "Parameter identification and backstepping control of uncertain Lü system," Chaos, Solitons \& Fractals, Vol. 18, pp 721-729.

[15] Wang, B. \& Wen, G. (2007) "On the synchronization of a class of chaotic systems based on backstepping method," Phys. Lett. A, Vol. 370, No. 1, pp 35-39.

[16] Gan, Q., Xu, R. \& Yang, P. (2012) "Synchronization of non-identical chaotic delayed fuzzy cellular neural networks based on sliding mode control," Comm. Nonlinear Sci. Numerical Simulation, Vol. 17, No. 1, pp 433-443.

[17] Sundarapandian, V. (2013) "Sliding mode controller design for the anti-synchronization of hyperchaotic Lü systems," International Journal of Cybernetics and Informatics, Vol. 2, No. 1, pp 3138.

[18] Sundarapandian, V. (2012) "Sliding controller design for the hybrid chaos synchronization of identical hyperchaotic Xu systems," International J. Instrumentation and Control Systems, Vol. 2, No. 4, pp 61-71.

[19] Yujun, N., Xingyuan, W., Mingjun, W. \& Huaguang, Z. (2010) "A new hyperchaotic system and its circuit implementation," Comm. Nonlinear Sci. Numerical Simulation, Vol. 15, pp 3518-3524.

[20] Hahn, W. (1967) The Stability of Motion, Springer Verlag, Berlin. 


\section{AUTHOR}

Dr. V. Sundarapandian graduated with D.Sc. degree in Electrical and Systems Engineering from Washington University, St. Louis, Missouri, USA in May 1996. He is working as Dean and Professor of the Research and Development Centre at Vel Tech University, Chennai, Tamil Nadu, India. Dr. Sundarapandian has published over 320 research articles in refereed international journals. He has also published 2 books with PHI, India and 1 book on Differential Equations with McGraw-Hill Education. He has published over 200 articles in National and International Conferences. He is the India Chair of AIRCC. He is the Editor-in-Chief of the five control journals - IJICS,

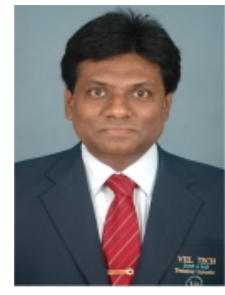

IJCTCM, IJITCA, IJCCMS and IJITMC - in AIRCC. His research interests are Control Systems, Chaos Theory, Intelligent Computing, Operations Research, Optimal Control, Scientific Modelling and Numerical Computing. He has delivered several Key Note Lectures on Control Systems, Chaos Theory and Scientific Computing. He has conducted many Workshops on Mathematical Modelling, Computational Science, Fuzzy Logic, Optimization, Operations Research, MATLAB and SCILAB. 